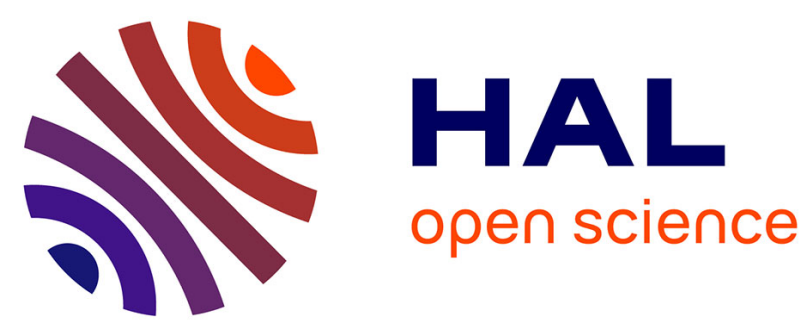

\title{
Etude expérimentale de l'interaction endommagement-température-état de contrainte-perméabilité du béton
}

Marta Choinska, Abdelhafid Khelidj, Frédéric Dufour, Gilles Pijaudier-Cabot

\section{- To cite this version:}

Marta Choinska, Abdelhafid Khelidj, Frédéric Dufour, Gilles Pijaudier-Cabot. Etude expérimentale de l'interaction endommagement-température-état de contrainte-perméabilité du béton. Revue Européenne de Génie Civil, 2007, 11 (6), pp.839-853. 10.1080/17747120.2007.9692963 . hal-01006818

\section{HAL Id: hal-01006818 https://hal.science/hal-01006818}

Submitted on 15 Mar 2017

HAL is a multi-disciplinary open access archive for the deposit and dissemination of scientific research documents, whether they are published or not. The documents may come from teaching and research institutions in France or abroad, or from public or private research centers.
L'archive ouverte pluridisciplinaire HAL, est destinée au dépôt et à la diffusion de documents scientifiques de niveau recherche, publiés ou non, émanant des établissements d'enseignement et de recherche français ou étrangers, des laboratoires publics ou privés. 


\title{
Etude expérimentale de l'interaction endommagement-température-état de contrainte-perméabilité du béton
}

\author{
Marta Choinska* - Abdelhafid Khelidj** \\ Frédéric Dufour* — Gilles Pijaudier-Cabot*
}

\author{
* R\&DO, GeM, UMR CNRS 6183, Ecole Centrale de Nantes \\ 1, rue de la Noë F-44321 Nantes cedex 3 \\ \{Marta.Choinska, Frederic.Dufour, Gilles.Pijaudier-Cabot\}@ec-nantes.fr \\ ** R\&DO, GeM, UMR CNRS 6183, IUT de Saint Nazaire \\ BP 420 Heinlex, F-44600 St Nazaire \\ Abdelhafid.Khelidj@iutsn.univ-nantes.fr
}

RÉSUMÉ. L'objectif de cette étude est de caractériser l'interaction entre endommagement, température et perméabilité du béton de structure. Les essais sont effectués sur des échantillons cylindriques creux, soumis à une compression uniaxiale et à des températures allant jusqu'à $150{ }^{\circ} \mathrm{C}$. Les résultats obtenus montrent que, pour un chargement inférieur à 80-85\% du chargement maximal, la variation de la perméabilité reste faible et peu influencée par le chargement. Quand le chargement excède 80-85\% du chargement maximal et approche le pic, l'endommagement croît rapidement, entraînant une augmentation importante de la perméabilité. Dans la phase post-pic, l'accroissement de la perméabilité devient très significatif suite à l'ouverture des macrofissures. L'augmentation de la perméabilité avec le chargement mécanique appliqué semble s'accroître à température élevée, provoquant l'altération de la structure poreuse du béton. Enfin, les résultats obtenus révèlent que les effets de l'endommagement et de la température peuvent être découplés pour l'estimation de l'évolution de la perméabilité du béton.

ABSTRACT. The objective of this study is to investigate damage-temperature-permeability interactions in structural concrete. The tests are performed on hollow cylindrical concrete specimens, subjected to compressive loading and temperature up to $150^{\circ} \mathrm{C}$. The results emphasize that at stress levels lower than 80-85\% of the peak stress, the variation of permeability is small and it is slightly influenced by the stress. As the load exceeds 80-85\% of the peak stress and approaches the peak, microcracking increases rapidly, causing an important increase of the permeability. In the post-peak phase the increase of permeability is much larger due to significant macrocrack width growth. The increase of permeability with the applied mechanical load seems to be greater with temperature, inducing the further alteration of concrete and dilation of the porous structure of the material. Finally, the obtained results reveal that the effects of damage and temperature may be decoupled for the estimation of the concrete permeability.

MOTS-CLÉS : béton, perméabilité, endommagement, fissuration, température.

KEYWORDS: concrete, permeability, damage, cracking, temperature. 


\section{Introduction}

La perméabilité du béton représente la capacité de ce matériau poreux à être traversé par un fluide sous un gradient de pression. Le flux se produit dans un réseau poreux, comportant des pores initiaux ainsi que dans des vides ou des fissures. Celles-ci, induites par diverses sollicitations, interconnectent généralement les chemins de percolation et diminuent ainsi la résistance du milieu au flux, ce qui provoque une augmentation de la perméabilité. La perméabilité est un paramètre pouvant influencer indirectement la durabilité des structures en béton en gouvernant la vitesse de pénétration d'agents agressifs, mais aussi directement la capacité de confinement lorsque la structure a un rôle d'étanchéité, ce qui est par exemple le cas des enceintes de confinement des centrales nucléaires.

Les données expérimentales existantes révèlent une certaine corrélation entre la perméabilité du béton et les effets des sollicitions mécaniques. Parmi ces données, certaines mettent en évidence une interaction entre l'endommagement (microfissuration diffuse) et la perméabilité, alors que d'autres soulignent une corrélation entre l'ouverture de fissure et la perméabilité. En effet, en fonction du régime de comportement mécanique (pré-pic ou post-pic), donc du niveau de fissuration (microfissuration diffuse ou macrofissuration localisée), l'interaction entre l'endommagement et la perméabilité se rapporte à une approche continue et l'interaction entre l'ouverture de fissure et la perméabilité est reliée à une approche discrète. Concernant l'approche continue, l'existence de l'interaction entre la perméabilité et l'endommagement diffus (microfissuration) a été prouvée théoriquement (Dormieux et Kondo, 2004 ; Chatzigeorgiou et al., 2005). D'une part, des résultats expérimentaux ont démontré une augmentation de la perméabilité au gaz du béton, mesurée sous une compression, à partir de 70-90 \% de la contrainte ultime (Sugiyama et al., 1996 ; Hearn et Lok, 1998). Ce niveau de contrainte correspond au développement et à l'interconnexion des microfissures dans la pâte de ciment. D'autre part, (Picandet et al., 2001) ont montré que la perméabilité résiduelle au gaz du béton augmente avec l'endommagement induit par la compression uniaxiale dans la phase pré-pic. Le modèle phénoménologique de (Picandet et al., 2001) a été corrélé avec les résultats expérimentaux pour des faibles endommagements, ceux-ci correspondant à une microfissuration diffuse dans le matériau. Concernant l'approche discrète, l'évolution de la perméabilité se base sur la loi d'écoulement de Poiseuille. Dans ce cas, la perméabilité d'une structure fissurée est fonction de la géométrie (ouverture, largeur, longueur) et de la topologie (connectivité, tortuosité) du réseau de fissures. Ces derniers paramètres ne sont pas accessibles dans les approches continues et pas toujours expérimentalement. Toutefois, des essais de traction par fendage, menés jusqu'à la rupture, ont mis en évidence l'évolution de la perméabilité à l'eau et au gaz avec l'ouverture de fissure (Wang et al., 1997 ; Picandet, 2001). Cependant, très peu d'études (Gérard, 1996) ont débouché sur une évolution de la perméabilité dans le régime de microfissuration diffuse et dans le régime de macrofissuration localisée jusqu'à la rupture en même temps, alors que l'évaluation de l'étanchéité d'une structure peut 
justement se rapporter à ces deux régimes de fissuration qui peuvent se succéder ou se superposer selon le type de chargement et selon la partie de structure considérée.

La température constitue un autre paramètre influençant la perméabilité du béton. Les mécanismes de l'interaction entre la température et la perméabilité sont étroitement liés avec des phénomènes au niveau microstructural apparaissant pendant la mise en température du béton. Parmi les plus importants figurent la modification de la teneur en eau due à l'évaporation de l'eau non liée jusqu'à $120{ }^{\circ} \mathrm{C}$ (Noumowé, 1995), la décomposition chimique de la pâte de ciment due à la perte de l'eau liée (déjà à partir de $105^{\circ} \mathrm{C}$ selon Bazant et Wittmann, 1982), la fissuration thermique provoquée par la dilatation thermique différentielle, la dilatation thermique libre de l'ensemble du matériau, le changement de pression interne des pores, etc. Etant liée à la dégradation de la microstructure du béton, la perméabilité du béton augmente avec la température. En particulier, la déshydratation chimique de la pâte de ciment modifie la porosité et la distribution de la taille des pores, et le comportement hydraulique du béton s'en trouve ainsi affecté (Tsimbrovska, 1998). Les résultats obtenus par (Tsimbrovska, 1998) ont montré que la perméabilité d'un mortier ou d'un béton subit une augmentation comprise entre un et deux ordres de grandeur à $400^{\circ} \mathrm{C}$ par rapport à $105^{\circ} \mathrm{C}$. (Lion et al., 2005) ont mis en évidence qu'un traitement thermique d'un mortier provoque une augmentation de la porosité et de la perméabilité au gaz, multipliant par sept sa valeur initiale (mesurée à $60^{\circ} \mathrm{C}$ ) après un traitement à $250^{\circ} \mathrm{C}$. Selon (Gawin et al., 2005), une relation type puissance entre la température et la perméabilité devrait être considérée. Cependant, certains auteurs (Gawin et al., 2002) utilisent l'endommagement afin de modéliser les effets de la température sur la perméabilité.

Les données expérimentales existantes révèlent ainsi une certaine corrélation entre la perméabilité et l'état mécanique du béton. Il en est de même pour l'influence de la température sur la perméabilité du béton, mais, quant aux effets couplés de la température et de l'état mécanique sur la perméabilité du béton, les données expérimentales sont éparses, voire inexistantes si on veut caractériser les deux régimes de fissuration (microfissuration diffuse ou macrofissuration localisée) sous charge et sous température. Ainsi, nous proposons de mener une étude sur un dispositif expérimental, développé dans notre laboratoire, qui permet de mesurer la perméabilité au gaz en fonction de la température allant jusqu'à $150{ }^{\circ} \mathrm{C}$ sous chargement mécanique dans les phases pré-pic et post-pic du chargement.

Le programme expérimental et les résultats sont exposés ci-dessous selon le plan suivant : dans un premier temps, le matériau étudié et le dispositif expérimental associé à la méthode d'essai pratiquée sont décrits, et dans un second temps, les résultats de l'étude expérimentale, à savoir l'évolution de la perméabilité avec le chargement mécanique et avec la température dans les phases pré-pic et post-pic, sont présentés et analysés. En conclusion, une loi d'évolution de la perméabilité en fonction de l'endommagement et de la température est proposée et commentée. 


\section{Matériau étudié}

\subsection{Composition}

Le béton utilisé dans cette étude expérimentale est un béton ordinaire de rapport Eau/Ciment $=0,6$ et de résistance à la compression de $26 \mathrm{MPa}$ à 28 jours. Le tableau 1 présente les éléments de la composition du béton. Le ciment utilisé est un ciment portland employé pour la construction des enceintes de confinement en France. Il est caractérisé par une faible chaleur d'hydratation et contient du filler calcaire. Les granulats employés sont les suivants: gravillon de type calcaire concassé et sable de Loire.

\begin{tabular}{|l|c|}
\hline \multicolumn{1}{|c|}{ Constituant } & Composition $\mathbf{~ e n ~} \mathbf{( k g / \mathbf { m } ^ { 3 } )}$ \\
\hline Gravillon calcaire, 4-12,5 mm & 939 \\
\hline Sable de Loire, 0-5 mm & 730 \\
\hline Ciment CEM II/A 42,5R PM-CP2 & 350 \\
\hline Eau totale & 210 \\
\hline Superplastifiant Glenium 21 & 1,1 \\
\hline Rapport eau/ciment & $\mathbf{0 , 6 0}$ \\
\hline
\end{tabular}

Tableau 1. Composition du béton

Les éprouvettes ont été confectionnées par trois gâchées d'environ 20 litres chacune. Dix-huit éprouvettes cylindriques, de géométrie donnée sur la figure 1, ont été coulées dans des moules métalliques avec des barres rondes métalliques de diamètre de $14 \mathrm{~mm}$, permettant d'obtenir ainsi la forme d'un cylindre troué. De plus, six éprouvettes de même dimensions que les éprouvettes trouées, mais pleines, ont été coulées dans des moules en carton.

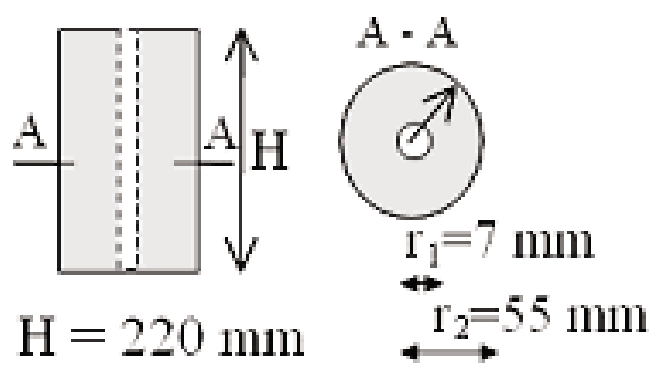

Figure 1. Géométrie de l'éprouvette 


\subsection{Cure appliquée}

Toutes les éprouvettes utilisées dans cette étude expérimentale ont suivi la même cure et le même conditionnement conformément aux recommandations de l'AFPCAFREM (1997). Après coulage, les éprouvettes ont été conservées pendant 24 heures en chambre humide à $20^{\circ} \mathrm{C}$ et $95 \%$ d'humidité relative. A l'issue de ce temps de conservation initiale, elles ont été démoulées, puis plongées dans l'eau à $20^{\circ} \mathrm{C}$ pendant 28 jours. Au cours de cette conservation, à environ 15 jours après le coulage, l'ensemble des éprouvettes a été rectifié en surface avec une précision de $0,1 \mathrm{~mm}$ à l'aide d'une meule équipée d'un disque diamant et lubrifiée à l'eau. A l'issue du temps de cure dans l'eau, les éprouvettes ont été séchées dans une étuve ventilée et régulée. Afin de diminuer les gradients hydriques et thermiques dans les éprouvettes, pouvant favoriser l'endommagement du béton, le séchage a été réalisé suivant deux étapes. D'abord, les éprouvettes ont été séchées à $80^{\circ} \mathrm{C}$ pendant 1 mois, puis à $105^{\circ} \mathrm{C}$ jusqu'à obtenir la masse constante. Suivant les recommandations de l'AFPC-AFREM (1997) la masse est considérée comme constante lorsque deux pesées espacées de 24 heures ne différent pas de plus de $0,05 \%$. Cependant, nous avons maintenu le séchage jusqu'à ce que la différence entre les pesées successives soit inférieure à $0,01 \%$. En conséquence, après le séchage pendant environ 2 mois à $105^{\circ} \mathrm{C}$, l'état de saturation du béton en eau a été proche de $0 \%$. Afin de maintenir cet état de saturation, la conservation finale jusqu'à l'essai de perméabilité a été effectuée dans une étuve ventilée et régulée à $40{ }^{\circ} \mathrm{C}$.

\subsection{Propriétés mécaniques}

Le tableau 2 présente les propriétés mécaniques du béton obtenues en compression à l'âge de 1 mois (après la cure humide), de 2 mois (après 1 mois de séchage à $80^{\circ} \mathrm{C}$ ) et de 4 mois (après 2 mois de séchage à $105^{\circ} \mathrm{C}$ ). Ce sont les propriétés moyennes, calculées sur trois éprouvettes à une échéance de mesure (deux cylindres troués et un cylindre plein).

\begin{tabular}{|l|c|c|l|}
\hline \multirow{2}{*}{\multicolumn{1}{|c|}{ Propriété }} & \multicolumn{3}{|c|}{ Age du béton (échéance de mesure) } \\
\cline { 2 - 4 } & $\begin{array}{l}\text { 1 mois (après cure } \\
\text { humide) }\end{array}$ & $\begin{array}{l}2 \text { mois (après } \\
\text { séchage à } 80^{\circ} \mathrm{C} \text { ) }\end{array}$ & $\begin{array}{l}4 \text { mois (après } \\
\text { séchage à } 105^{\circ} \mathrm{C} \text { ) }\end{array}$ \\
\hline Module d'élasticité $(\mathrm{GPa})$ & 23 & 22 & 16 \\
\hline Résistance $(\mathrm{MPa})$ & 26 & 32 & 28 \\
\hline $\begin{array}{l}\text { Déformation au pic de contrainte } \\
(-)\end{array}$ & $2,2.10^{-03}$ & $2,5.10^{-03}$ & $2,6.10^{-03}$ \\
\hline
\end{tabular}

Tableau 2. Propriétés mécaniques du béton calculées sur trois éprouvettes 
Nous remarquons que les propriétés mécaniques du béton évoluent dans le temps avec les conditions de conservation. En particulier, avec le séchage, la résistance augmente et le module d'élasticité diminue dû à l'endommagement induit par la température de séchage (Hearn, 1999 ; Burlion et al., 2005).

\section{Dispositifs expérimentaux et procédure d'essai}

\subsection{Dispositif d'application de sollicitations mécaniques et thermiques}

Les sollicitations mécaniques sont appliquées à l'aide d'une presse hydraulique asservie en déplacement. L'éprouvette, sollicitée en compression uniaxiale, est placée entre deux plateaux métalliques spécialement usinés afin de transmettre l'effort de compression et de permettre en même temps l'injection du fluide percolant. Les déplacements axiaux de l'éprouvette sont mesurés pendant l'essai dans sa partie médiane (longueur de base : $105 \mathrm{~mm}$ ) par trois capteurs LVDT disposés à $120^{\circ}$ sur la cellule extensométrique. L'éprouvette instrumentée et sollicitée mécaniquement est placée dans une enceinte climatique à température et humidité relative contrôlées. Par souci de simplification, nous limitons l'étude aux matériaux secs; c'est pourquoi l'humidité relative générée par l'enceinte est constamment maintenue la plus proche possible de $0 \%$ (l'erreur maximale par rapport à la consigne est de $\pm 5 \%$ ). Cependant, les essais à des niveaux de saturation variables peuvent également être menés.

\subsection{Dispositif de mesure de la perméabilité}

Le schéma de principe du fonctionnement du dispositif de mesure de la perméabilité est montré sur la figure 2 .

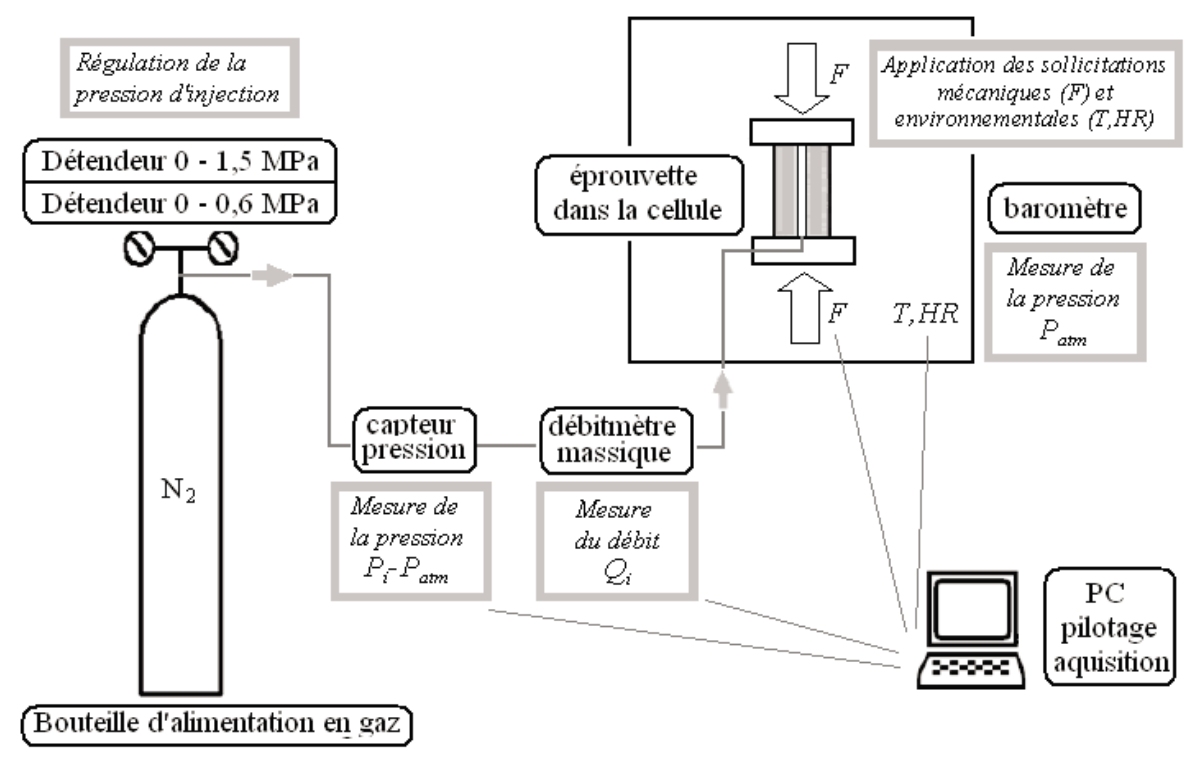

Figure 2. Schéma du dispositif de mesure de la perméabilité au gaz 
La méthode de mesure de la perméabilité au gaz consiste à appliquer une pression relative constante du gaz $\Delta P$ (différence entre la pression injectée absolue $P_{i}$ et la pression atmosphérique $P_{a t m}$ ) jusqu'à la stabilisation de l'écoulement du gaz à travers le matériau (régime permanent). Le gaz utilisé est de l'azote $\left(\mathrm{N}_{2}\right) \mathrm{sec}$, car il reste inerte vis-à-vis des constituants du béton, contrairement à l'eau. L'éprouvette est placée dans la cellule du perméamètre constituée d'un système à deux plateaux métalliques. Par l'intermédiaire d'un plateau métallique percé, le gaz est injecté à partir de la bouteille d'alimentation au niveau de la surface inférieure de l'échantillon à une pression $\mathrm{P}_{\mathrm{i}}$ (régulée par le régulateur maintenant le niveau de pression, puis mesurée par le capteur de pression). La pression atmosphérique est mesurée au cours de l'essai par un baromètre digital. Etant donné que les deux faces inférieure et supérieure de l'éprouvette sont rendues étanches, l'écoulement obtenu est radial. Le débit du gaz est mesuré en amont de l'éprouvette par un débitmètre massique qui convertit le débit massique en un débit volumique équivalent.

Dans le cas d'écoulement d'un gaz, fluide compressible, les vitesses d'écoulement et le débit volumique varient en tout point avec la pression. Le débit massique reste toutefois constant. L'application du principe de conservation de la masse permet d'utiliser ainsi la loi de Darcy pour l'écoulement isotherme d'un gaz considéré comme parfait. Selon le principe de conservation de la masse, la perméabilité $k_{a}\left[\mathrm{~m}^{2}\right]$ peut être ainsi déterminée à partir du débit et de la pression en amont de l'écoulement :

$$
k_{a}=\frac{Q_{i} \cdot P_{i} \cdot \mu \cdot \ln \left(\frac{r_{2}}{r_{1}}\right)}{\pi \cdot H \cdot\left(P_{i}^{2}-P_{a t m}^{2}\right)}
$$

où $Q_{i}\left[\mathrm{~m}^{3} / \mathrm{s}\right]$ est le débit volumique de gaz à pression d'injection, $P_{i}[\mathrm{~Pa}]$ est la pression absolue d'injection, $\mu$ [Pa.s] est la viscosité dynamique de l'azote, $P_{\text {atm }}[\mathrm{Pa}]$ est la pression atmosphérique, et $r_{1}, r_{2}, H[\mathrm{~m}]$ sont respectivement le rayon intérieur, le rayon extérieur et la hauteur de l'éprouvette cylindrique. La perméabilité $k_{a}$ dépend de la pression d'injection; elle n'est pas alors une caractéristique intrinsèque du matériau. Cependant, avec l'approche de (Klinkenberg, 1941), la perméabilité intrinsèque, due uniquement à l'écoulement visqueux, peut être déterminée à partir de plusieurs mesures de perméabilité apparente $k_{a}$ pour différentes pressions d'injection $P_{i}$.

\subsection{Procédure expérimentale pour mesurer la perméabilité sous chargement et sous température}

La procédure d'essai pour une éprouvette est la suivante : premièrement, la perméabilité initiale à $20^{\circ} \mathrm{C}$ est déterminée. La suite de l'essai comprend la mise en température de l'éprouvette dans l'enceinte climatique à $20^{\circ} \mathrm{C}, 105^{\circ} \mathrm{C}$ ou $150{ }^{\circ} \mathrm{C}$. 
Après le chauffage à $105^{\circ} \mathrm{C}$ ou $150^{\circ} \mathrm{C}$ pendant 60 heures, durant lequel la stabilisation de la température à travers l'éprouvette est atteinte, la perméabilité, influencée uniquement par la température et appelée perméabilité de référence, est mesurée (dans le cas d'essai à $20{ }^{\circ} \mathrm{C}$ la perméabilité de référence est égale à la perméabilité initiale). Ensuite, les mesures de la perméabilité sont effectuées sous la température imposée précédemment et à différents niveaux du chargement mécanique. Après chaque chargement, l'éprouvette est déchargée et la mesure de la perméabilité est à nouveau effectuée.

Tous les résultats présentés ci-dessous se rapportent aux valeurs de la perméabilité intrinsèque, obtenues au minimum avec trois échantillons par température.

\section{Résultats}

La perméabilité initiale du béton étudié varie selon les gâchées et les éprouvettes sur une plage située entre $6.10^{-17} \mathrm{~m}^{2}$ et $12.10^{-17} \mathrm{~m}^{2}$. Cet écart illustre une bonne reproductibilité du matériau. Cependant, la perméabilité initiale du béton étudié est supposée supérieure à celle d'un béton d'ouvrage pour deux raisons. La première raison concerne le taux de saturation, qui est plus élevé dans un béton d'ouvrage. La deuxième est liée au processus expérimental du séchage qui peut entraîner un endommagement par effets thermiques (voir, par exemple, Hearn, 1999). Néanmoins, l'ensemble des éprouvettes considérées dans cette étude a le même taux de saturation, ainsi que le même endommagement de séchage (état initial). Etant donné que nous nous intéressons plus particulièrement à la variation de perméabilité, plutôt qu'à sa valeur absolue, la perméabilité initiale de chaque éprouvette est considérée pour une valeur de référence dans l'analyse des résultats présentés par la suite.

\subsection{Influence de l'état de contrainte sur la perméabilité}

Afin de mettre en évidence l'influence de l'état de contrainte sur la perméabilité, nous l'avons mesurée sous chargement et après déchargement pour différents niveaux de chargement dans les phases pré-pic et post-pic du comportement mécanique. Le rapport entre ces deux perméabilités en fonction de la déformation axiale relative maximale, appliquée au cours du chargement, est montré sur la figure 3. Ce rapport n'est ni constant ni linéaire et varie avec le chargement appliqué. Pour les trois températures considérées, il atteint son maximum, compris entre 2,5 et 3, juste au début de la phase post-pic pour la déformation axiale avoisinant $110 \%-120 \%$ de la déformation au pic de contrainte. Il s'accroît avec la température car la différence entre la perméabilité sous chargement et après déchargement devient plus prononcée sous température élevée, puis tend à devenir constante dans la phase post-pic. 


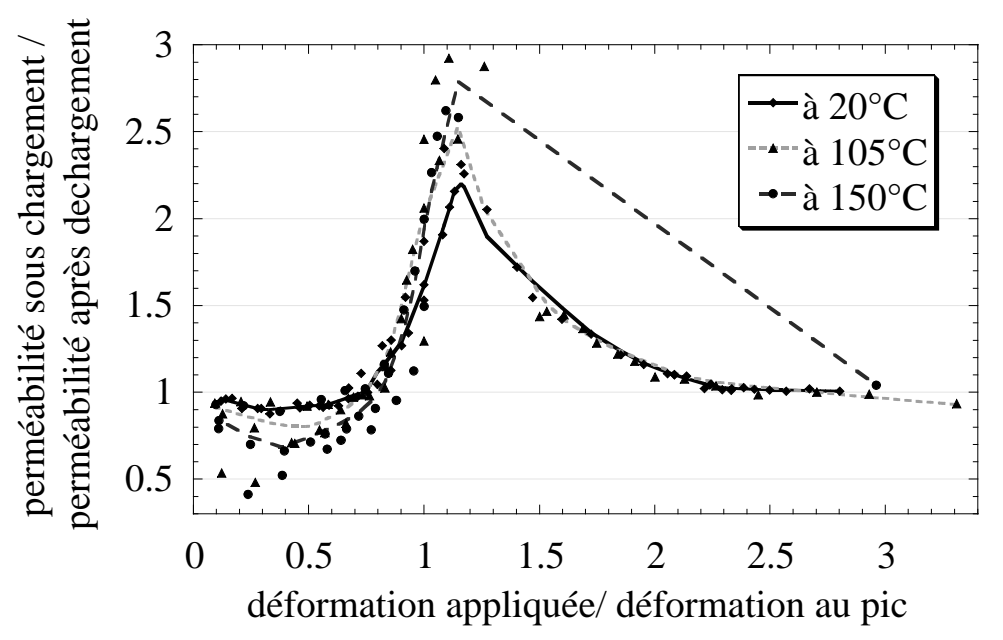

Figure 3. Evolution du rapport entre la perméabilité sous chargement et après déchargement en fonction de la déformation axiale relative maximale appliquée pour trois températures considérées (les lignes correspondent aux courbes d'ajustement des données expérimentales)

Les résultats obtenus montrent que la perméabilité sous chargement est initialement plus faible que la perméabilité mesurée après déchargement. Le chargement agit sur la fermeture des microfissures existantes dans le matériau (voir figure 4). Quand le chargement excède $75-80 \%$ du chargement maximal et approche le pic, un réseau de microfissures interconnectées dans la pâte de ciment commence à se propager. En effet, une différence importante entre la perméabilité mesurée sous chargement et après déchargement apparaît, la première devenant plus grande que la dernière.

Finalement, l'évolution montrée sur la figure 3 révèle la capacité du matériau à rétablir la perméabilité suite à la refermeture des fissures après déchargement. Cette capacité est significative dans la phase pré-pic et au début de la phase post-pic, puis décroît fortement dans la phase post-pic où la refermeture des fissures ne devient que partielle (voir impossible) suite aux glissements des lèvres de fissure en cisaillement et au phénomène de crushing.

\subsection{Evolution de la perméabilité avec le chargement dans la phase pré-pic}

La figure 4 montre l'évolution de la perméabilité relative avec la contrainte subie au cours du chargement dans la phase pré-pic du comportement mécanique. La perméabilité relative représente le rapport entre la perméabilité sous chargement mécanique et sous la température de $20^{\circ} \mathrm{C}, 105^{\circ} \mathrm{C}$ ou $150{ }^{\circ} \mathrm{C}$, et la perméabilité de référence déterminée à la même température mais sans chargement. 


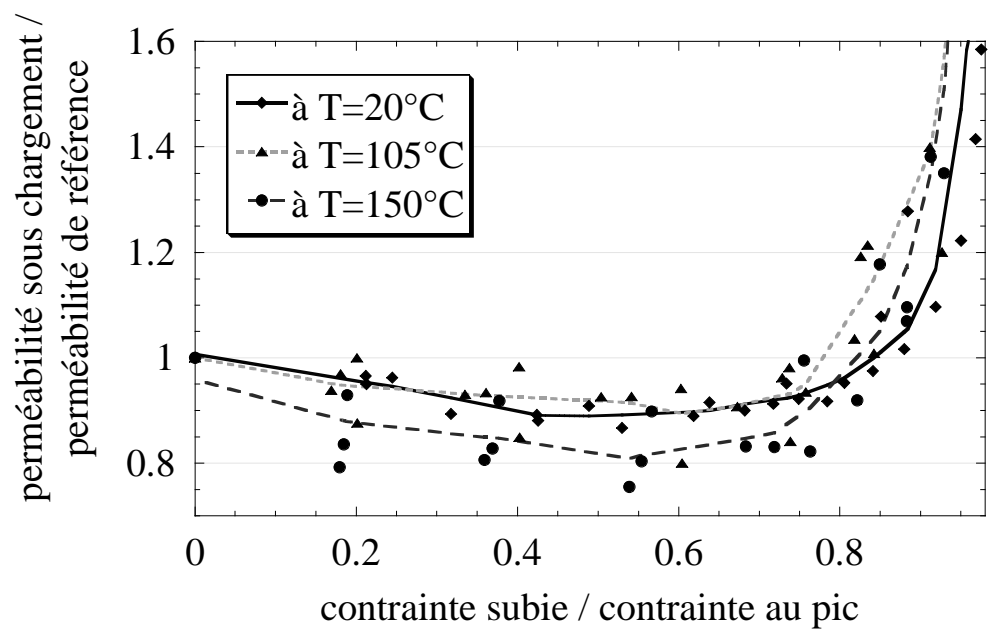

Figure 4. Evolution de la perméabilité relative, déterminée sous chargement et après déchargement, avec la contrainte relative (maximale) subie au cours du chargement pour les trois températures considérées (les lignes correspondent aux courbes d'ajustement des données expérimentales)

Les résultats obtenus révèlent des phases caractéristiques de l'évolution de la perméabilité avec la contrainte dans la phase pré-pic. La présence de ces phases reste indépendante de la température. La première phase, montrant une légère diminution de la perméabilité, correspond à une plage de contrainte entre $0 \%$ et 40 $60 \%$ de la contrainte maximale. La deuxième phase représente une légère diminution de la perméabilité entre $40-60 \%$ et $80-85 \%$ de la contrainte maximale. La troisième phase se distingue à partir de $80-85 \%$ de la contrainte maximale lorsque la perméabilité augmente nettement.

La présence de ces phases s'explique par les modifications de la structure poreuse du béton sous la compression uniaxiale, qui sont reliées au comportement volumique du béton (Sugiyama et al., 1996 ; Choinska, 2006). Ce comportement est d'abord contractant (jusqu'à 40-60\% de la contrainte maximale), puis finalement dilatant (à partir de 80-85\% de la contrainte maximale) lorsque les microfissures orientées se propagent à travers la pâte de ciment.

\subsection{Evolution de la perméabilité avec le chargement jusqu'à la phase post-pic}

Les résultats présentés dans la figure 5 montrent l'évolution totale de la perméabilité relative (rapport entre la perméabilité à $20^{\circ} \mathrm{C}, 105^{\circ} \mathrm{C}$ ou $150{ }^{\circ} \mathrm{C}$ et la perméabilité initiale à $20^{\circ} \mathrm{C}$ ) sous chargement et après déchargement en fonction de la déformation relative maximale appliquée pour les trois températures considérées.

Nous observons que les perméabilités deviennent plus importantes avec la température. Ce phénomène peut s'expliquer par la température qui rend le comportement du béton plus fragile. En effet, lorsque l'essai est réalisé sous $20^{\circ} \mathrm{C}$, le réseau de plusieurs macrofissures, parallèles au chargement, est facilement 
discernable à la rupture qui est plutôt ductile. Cependant, sous $150{ }^{\circ} \mathrm{C}$, une seule macrofissure traverse généralement l'éprouvette et la rupture est brutale. De plus, la formation nette d'un plan de cisaillement est remarquée. Sous $105^{\circ} \mathrm{C}$, les deux modes de rupture ont été observés de manière aléatoire. Néanmoins, dans la plupart des cas (trois fois sur quatre), la rupture a été plus proche de celle observée sous $20{ }^{\circ} \mathrm{C}$. Ces observations prouvent que la microfissuration diffuse sous une température élevée est moins développée, que des macrofissures traversantes apparaissent plus tôt et qu'elles ont des ouvertures plus importantes qu'à $20^{\circ} \mathrm{C}$. En conséquence, selon la loi de Poiseuille, la perméabilité (ou plutôt le flux) du matériau avec des grandes macrofissures (sous une température élevée) devient plus importante que celle du matériau comportant des microfissures ou macrofissures plus fines (sous $20^{\circ} \mathrm{C}$ ).

Au-delà, nous notons que la cinétique d'évolution de la perméabilité augmente également avec la température. A $105^{\circ} \mathrm{C}$, bien que cette température ait été appliquée à tous les spécimens pendant le séchage, la cinétique de la perméabilité est plus grande qu'à $20^{\circ} \mathrm{C}$. A $150^{\circ} \mathrm{C}$, la perméabilité n'a pu être mesurée en continu dans la phase post-pic en raison de la fragilité du béton. En effet, les mesures de la perméabilité ont été effectuées jusqu'au pic, puis à la rupture. Nous avons interpolé ces résultats par une courbe d'ajustement (lissage) entre le pic et la rupture, en supposant que sa forme ne devrait pas être très différente de celle observée à d'autres températures, notamment à $105^{\circ} \mathrm{C}$ et $20^{\circ} \mathrm{C}$. En conséquence, nous observons que cette cinétique de l'évolution de la perméabilité à $150^{\circ} \mathrm{C}$ est plus grande qu'à $20^{\circ} \mathrm{C}$ et $105^{\circ} \mathrm{C}$. Toutefois, nous observons que dans des conditions d'essai particulières (béton séché à $105^{\circ} \mathrm{C}$, température d'essai $150{ }^{\circ} \mathrm{C}$ au maximum), les effets du chargement mécanique sont prépondérants par rapport à ceux de la température.

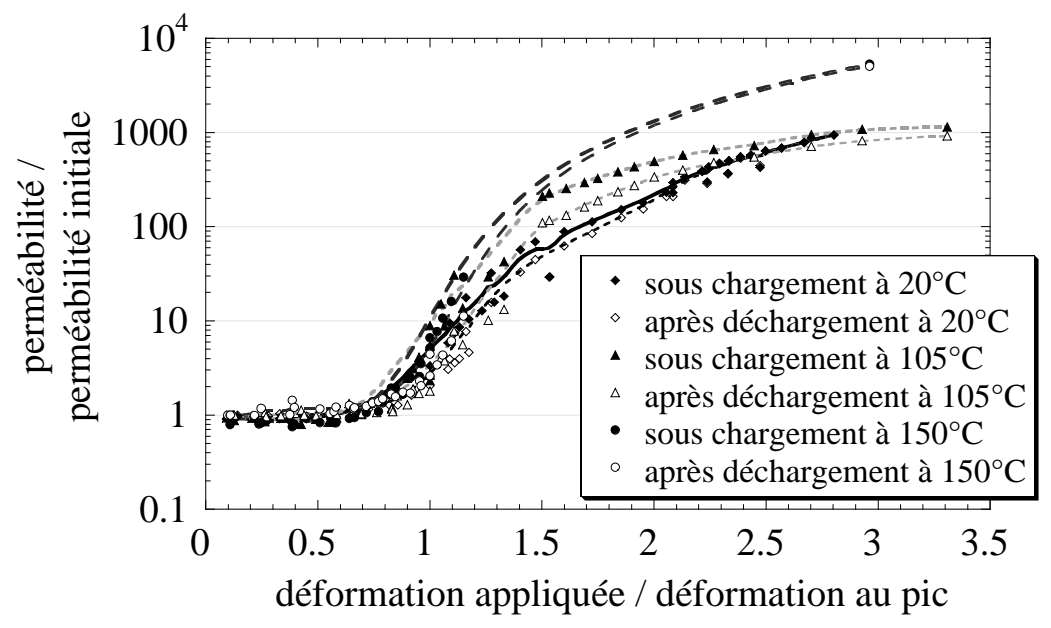

Figure 5. Evolution totale de la perméabilité relative (sous chargement et après déchargement) avec la déformation relative maximale pour trois températures (échelle logarithmique pour la perméabilité, les lignes correspondent aux courbes d'ajustement des données expérimentales) 
Les évolutions de la perméabilité, obtenues à $20{ }^{\circ} \mathrm{C}, 105{ }^{\circ} \mathrm{C}$ et $150{ }^{\circ} \mathrm{C}$, soulignent la présence de plusieurs régimes d'évolution de la perméabilité avec la déformation axiale. Les tendances observées sont en accord avec celles des résultats expérimentaux obtenus par (Wang et al., 1997; Gérard, 1996). Ces régimes apparaissent indépendamment de la température et de l'état de contrainte et ils sont reliés à la création des microfissures diffuses dans la phase pré-pic qui, par coalescence, se localisent pour former des fissures localisées macroscopiques se propageant dans la phase post-pic.

Nous supposons que, suite à la localisation de la fissuration et la formation des macrofissures traversantes, le flux à travers le milieu fissuré devient tributaire de la macrofissuration, tout en étant négligeable dans les zones microfissurées. En effet, suite à la localisation de la fissuration, la localisation du flux se produit. En conséquence, la perméabilité du milieu fissuré dans ce régime devient une fonction de l'ouverture des fissures selon la loi de Poiseuille.

\section{Relation endommagement-température-perméabilité}

Etant donné que la température à de faibles niveaux (tels que $150{ }^{\circ} \mathrm{C}$ ) provoque plutôt un endommagement diffus (microfissuration à l'interface pâte-granulat) et que la localisation de la fissuration a lieu juste avant le pic de contrainte, nous proposons d'établir, sur la base de nos résultats expérimentaux, une relation entre les effets de la température et de l'endommagement diffus sur la perméabilité. Comme l'effet de l'état de contrainte peut être découplé de la relation de l'évolution de la perméabilité, son influence n'est pas considérée dans cette analyse. Seule l'évolution de la perméabilité après déchargement est donc prise en compte.

L'endommagement est déterminé à partir de la variation du module d'élasticité $\mathrm{du}$ déchargement. L'endommagement thermique, existant pourtant à $150^{\circ} \mathrm{C}$ (Choinska, 2006), est difficilement quantifiable à partir de la variation du module d'élasticité du déchargement en compression. Cela peut être attribué au fait que l'orientation des fissures n'est pas vraiment la même sous chargement en compression et sous température. En effet, nous n'identifions pas d'endommagement sous un chargement uniaxial, alors qu'un chargement isotrope triaxial pourrait peut-être le mettre en évidence. Toutefois, dans le cas de nos essais, l'endommagement thermique est nettement plus faible que celui dû au chargement mécanique. Par conséquent, l'endommagement calculé correspond uniquement à un endommagement mécanique. Sur la figure $6 \mathrm{a}$, les évolutions de la perméabilité relative en fonction de l'endommagement calculé pour chaque éprouvette sous température de $20{ }^{\circ} \mathrm{C}, 105{ }^{\circ} \mathrm{C}$ ou $150{ }^{\circ} \mathrm{C}$ sont présentées. La perméabilité relative à l'état mécanique est obtenue comme le rapport entre la perméabilité après déchargement et la perméabilité de référence à la même température. Nous observons que les évolutions de ces perméabilités relatives, déterminées pour différentes températures, suivent une même évolution représentée par la courbe 
principale sur la figure 6a. Cette évolution se révèle donc être indépendante de la température. Quant aux effets de la température sur la perméabilité (figure 6b), l'évolution de la perméabilité de référence normalisée par rapport à la perméabilité initiale pour différents niveaux du chargement reste indépendante de l'endommagement.

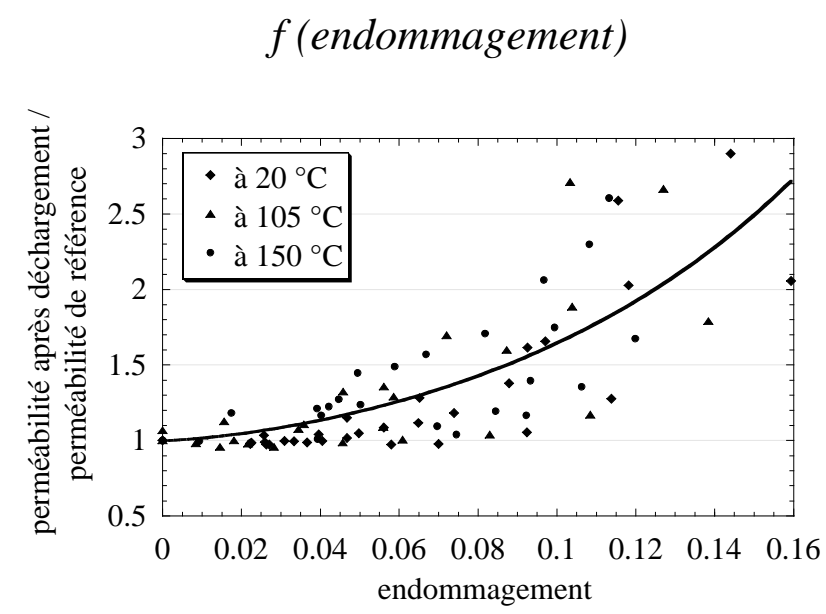

(a)

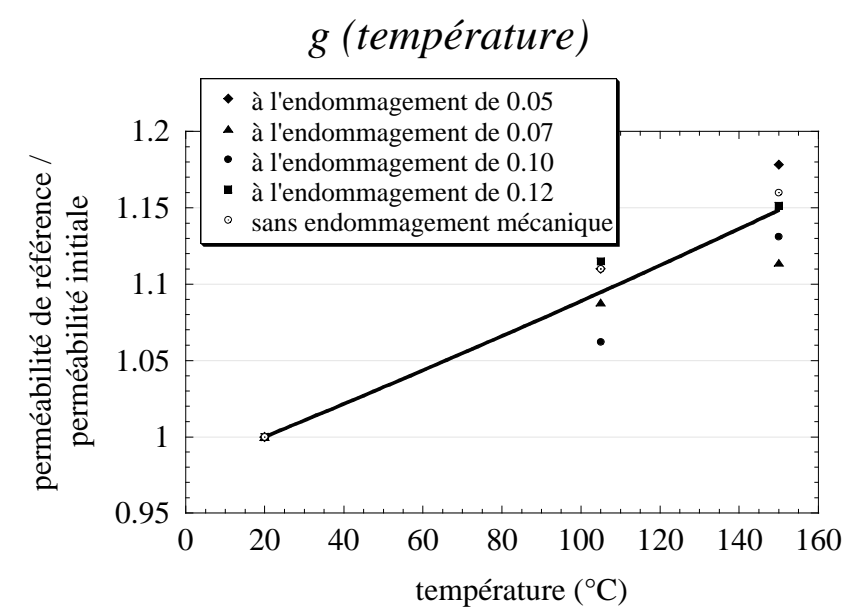

(b)

Figure 6. Evolution des perméabilités relatives avec (a) l'endommagement et (b) la température (les lignes correspondent aux courbes d'ajustement des données expérimentales)

L'identification de deux évolutions indépendantes montrées respectivement sur les figures $6 \mathrm{a}$ et $6 \mathrm{~b}$, nous a permis de proposer une loi générique d'évolution de la perméabilité $: k=f$ (endommagement) $g$ (température), où $k$ est la perméabilité, $k_{0}$ la perméabilité initiale et les fonctions $f$ et $g$ représentent respectivement les relations présentées sur les figures 6a et 6b (Choinska et al., 2007). Par ailleurs, le format de ce modèle d'interaction, ajusté sur nos résultats expérimentaux, est en accord avec celui proposé par (Gawin et al., 2002).

\section{Conclusion}

Cette étude a permis de caractériser les effets du chargement mécanique et de la température sur la perméabilité du béton. Les résultats apportés dans cette étude amènent les conclusions suivantes :

- dans la phase pré-pic, qui reproduit le comportement du béton de structure dans les cas de chargement de service, plusieurs phases d'évolution de la perméabilité apparaissent indépendamment de la température du béton. Suite au changement du comportement volumique du béton sous la compression uniaxiale, une légère diminution, suivie par une légère augmentation de la perméabilité est observée. Cette phase correspond à la refermeture des microfissures préexistantes, puis à la création probable de nouvelles microfissures qui ne sont pas encore 
interconnectées. A partir de $80-85 \%$ du chargement au pic, une nette augmentation de la perméabilité, déterminée sous chargement et après déchargement, est constatée. Elle est attribuée probablement à l'apparition de microfissures continues à travers la pâte de ciment. Cette augmentation s'intensifie avec l'augmentation de la température ;

- dans la phase post-pic, qui peut reproduire le comportement du béton de structure dans les cas de chargement exceptionnel (par exemple accident sur une enceinte), la perméabilité augmente significativement en raison de la croissance de l'ouverture et de la largeur des fissures macroscopiques. Ainsi, le flux à travers le milieu fissuré devient tributaire de la macrofissuration, tout en étant négligeable dans les zones microfissurées. En effet, suite à la localisation de la fissuration, la localisation du flux se produit ;

- les effets de l'endommagement et de la température peuvent être découplés pour l'estimation de l'évolution de la perméabilité. Toutefois, la validation de la loi d'interaction proposée pour d'autres températures et sur d'autres bétons paraît nécessaire. Par ailleurs, il serait intéressant de la vérifier non seulement sur un matériau sec mais également sur un matériau avec un niveau de saturation variable. Cependant, cette loi d'évolution ne peut s'appliquer que lorsque le matériau est endommagé de façon homogène, donc avant la localisation des déformations. En effet, elle n'est valable qu'avant le pic de contrainte, donc pour des endommagements modérés. Afin de dépasser cette limite pour pouvoir également modéliser l'accroissement de la perméabilité avec la fissuration localisée en phase post-pic, un autre paramètre comme l'ouverture de fissure devrait être pris en compte dans cette loi d'évolution. L'intégration de ce paramètre demande d'abord d'établir une équivalence entre le champ d'endommagement et l'ouverture de fissure (Choinska, 2006). Une fois déterminée l'ouverture de fissure équivalente au champ d'endommagement, la loi de Poiseuille pourra être appliquée pour estimer le débit de fluide d'une structure à la rupture.

\section{Bibliographie}

AFPC-AFREM, Extrait du compte rendu des Journées Techniques de l'AFPC-AFREM, Durabilité des bétons, Méthodes recommandés pour la mesure des grandeurs associées à la durabilité, Mode opératoire recommandé, essai de perméabilité au gaz du béton durci, 11 et 12 décembre 1997, Toulouse.

Bazant Z.P., Wittmann F.H., Creep and shrinkage in concrete structures, John Wiley \& Sons, 1982.

Burlion N., Bourgeois F., Shao J.-F., "Effects of desiccation on mechanical behaviour of concrete", Cement and Concrete Research, vol. 27, 2005, p. 367-379.

Chatzigeorgiou G., Picandet V., Khelidj A., Pijaudier-Cabot G., "Coupling between progressive damage and permeability of concrete: analysis with a discrete model", Int. J. Anal. Meth. Geomech., vol; 29, 2005, p. 1005-1018. 
Choinska M., Khelidj A., Chatzigeorgiou G., Pijaudier-Cabot G., "Effects and Interactions of Temperature and Stress-level Related Damage on Permeability of Concrete", Cement and Concrete Research, vol. 37, 2007, p. 79-88.

Choinska M., Effets de la température, du chargement mécanique et de leurs interactions sur la perméabilité du béton de structure, Thèse de Doctorat, Ecole Centrale de Nantes et Université de Nantes, 2006.

Dormieux L., Kondo D., «Approche micromécanique du couplage perméabilitéendommagement », C.R. Mécanique, 332, 2004, p. 135-140.

Gawin D., Alonso C., Andrade C., Majorana C.E., Pesavento F., "Effect of damage on permeability and hygro-thermel behaviour of HPCs at elevated temperatures: Part 1. Experimental results", Computers and Concrete, 2005, p. 189-202.

Gawin D., Pesavento F., Schlefler B.A., "Simulation of damage-permeability coupling in hygro-thermo-mechanical analysis of concrete at high temperature", Comm. in Num. Meth. Engrg. 18, 2002, p. 113-119.

Gérard B., Contribution des couplages mécanique-chimie-transfert dans la tenue à long terme des ouvrages de stockage de déchets radioactifs, Thèse de doctorat, ENS Cachan, 1996.

Hearn N., Lok G., "Measurement of Permeability under Uniaxial Compression-A Test Method", ACI Materials Journal, 95, 1998, p. 691-694.

Hearn N., "Effect of shrinkage and load-induced cracking on water permeability of concrete", ACI Materials Journal, 96, 1999, p. 234-241.

Klinkenberg L.J., "The permeability of porous media to liquids and gases", American Petroleum Institute, Drilling and Production Practice, 1941, p. 200-213.

Lion M., Skoczylas F., Lafhaj Z., Sersar M., "Experimental study on a mortar, Temperature effects on porosity and permeability, Residual properties or direct measurements under temperature", Cement and Concrete Research, 35, 2005, p. 1937-1942.

Noumowé A., Effet de hautes températures $\left(20-600{ }^{\circ} \mathrm{C}\right)$ sur le béton. Cas particulier du béton à hautes performance, Thèse de Doctorat, INSA Lyon, 1995.

Picandet V., Khelidj A., Bastian G., "Effect of axial compressive damage on gas permeability of ordinary and high-performance concrete", Cement and Concrete Research, 31, 2001, p. $1525-1532$.

Picandet V., Influence d'un endommagement mécanique sur la perméabilité et sur la diffusivité hydrique des bétons, Thèse de Doctorat, Université de Nantes, 2001.

Sugiyama T., Bremner T.W., Holm T.A., "Effect of Stress on Gas Permeability in Concrete", ACI Materials Journal, 93, 1996, p. 443-450.

Tsimbrovska M., Dégradation des bétons à hautes performances soumis à des températures élevées. Evolution de la perméabilité en liaison avec la microstructure, Thèse de Doctorat, Université Grenoble 1, 1998.

Wang K., Jansen D.C., Shah S.P., Karr A.F., "Permeability study of cracked concrete", Cement and Concrete Research, 27, 1997, p. 381-393. 